\title{
Nickel-Catalyzed Site- and Stereoselective Reductive Alkylalkynylation of Alkynes
}

\author{
Yi Jiang ${ }^{1}$, Jiaoting Pan $^{1,2}$, Tao Yang ${ }^{1}$, Joel Jun Han $\mathrm{Lim}^{1}$, Yu Zhao ${ }^{1,2 *} \&$ Ming Joo Koh ${ }^{1 *}$
}

Development of a catalytic multicomponent reaction by orthogonal activation of readily available substrates for the streamlined difunctionalization of alkynes is a compelling objective in organic chemistry. Alkyne carboalkynylation, in particular, offers a direct entry to valuable 1,3-enynes with different substitution patterns. Here, we show that the synthesis of stereodefined 1,3-enynes featuring a trisubstituted olefin is achieved by merging alkynes, alkynyl bromides and redox-active $\mathbf{N}$-(acyloxy)phthalimides through nickel-catalyzed reductive alkylalkynylation. Products are generated in up to $89 \%$ yield as single regio- and $E$ isomers. Transformations are tolerant of diverse functional groups and the resulting 1,3-enynes are amenable to further elaboration to synthetically useful building blocks. With olefin-tethered $\boldsymbol{N}$-(acyloxy)phthalimides, a cascade radical addition/cyclization/alkynylation process can be implemented to obtain 1,5-enynes. The present study underscores the crucial role of redoxactive esters as superior alkyl group donors compared to haloalkanes in reductive alkyne dicarbofunctionalizations.

Aliphatic carboxylic acids are abundant feedstock chemicals that have found extensive utility in chemical synthesis. ${ }^{1-2}$ With recent advances in cross-coupling chemistry, these readily available organic molecules which were once regarded as non-traditional cross-partners, have emerged as convenient alkyl donors in catalytic decarboxylative $\mathrm{C}-\mathrm{C}$ bond forming transformations, either via the innate carboxyl groups ${ }^{3-10}$ or their activated ester derivatives. ${ }^{11-23}$ These developments are further driven by the much wider commercial availability of alkyl carboxylic acids as compared to conventional alkyl halides or alkylmetal reagents. ${ }^{12,22,24}$ A related class of reactions that utilize $N$-(acyloxy)phthalimides (or NHPI esters) involve decarboxylative alkyl additions to alkynes ${ }^{25}$ or alkenes. ${ }^{26-37}$ Intrigued by previous studies, we speculated if alkyl NHPI esters could be exploited in three-component processes by merging with an alkyne and an alkynyl halide to deliver synthetically valuable acyclic 1,3-enyne motifs, conjugated entities commonly embedded within natural products, pharmaceuticals, agrochemicals and materials. ${ }^{38-42}$

\footnotetext{
${ }^{1}$ Department of Chemistry, National University of Singapore, 12 Science Drive 2, Republic of Singapore, 117549.

2 Joint School of National University of Singapore and Tianjin University, International Campus of Tianjin University, Binhai New City, Fuzhou 350207, China.
} 
Various routes to architecturally analogous 1,3-enynes that contain a trisubstituted alkene moiety ${ }^{43-54}$ have been developed, but the majority focused on two-component systems involving coupling reactions of elaborated alkynes/alkenes as starting materials. ${ }^{43,47-48,51-54}$ Threecomponent catalytic regimes ${ }^{44-46,49}$ starting from simpler, more readily accessible substrates offer a more practical approach to expeditiously assemble the desired products. However, these methods suffer from a number of shortcomings. Activated $\alpha$-functionalized alkyl halides are frequently employed to generate a stabilized alkyl radical species for alkyne addition, ${ }^{44}$ and a second catalyst is sometimes required to promote $C(s p)-C\left(s p^{2}\right)$ bond formation ${ }^{44-45,49}$ which limit broad utility.

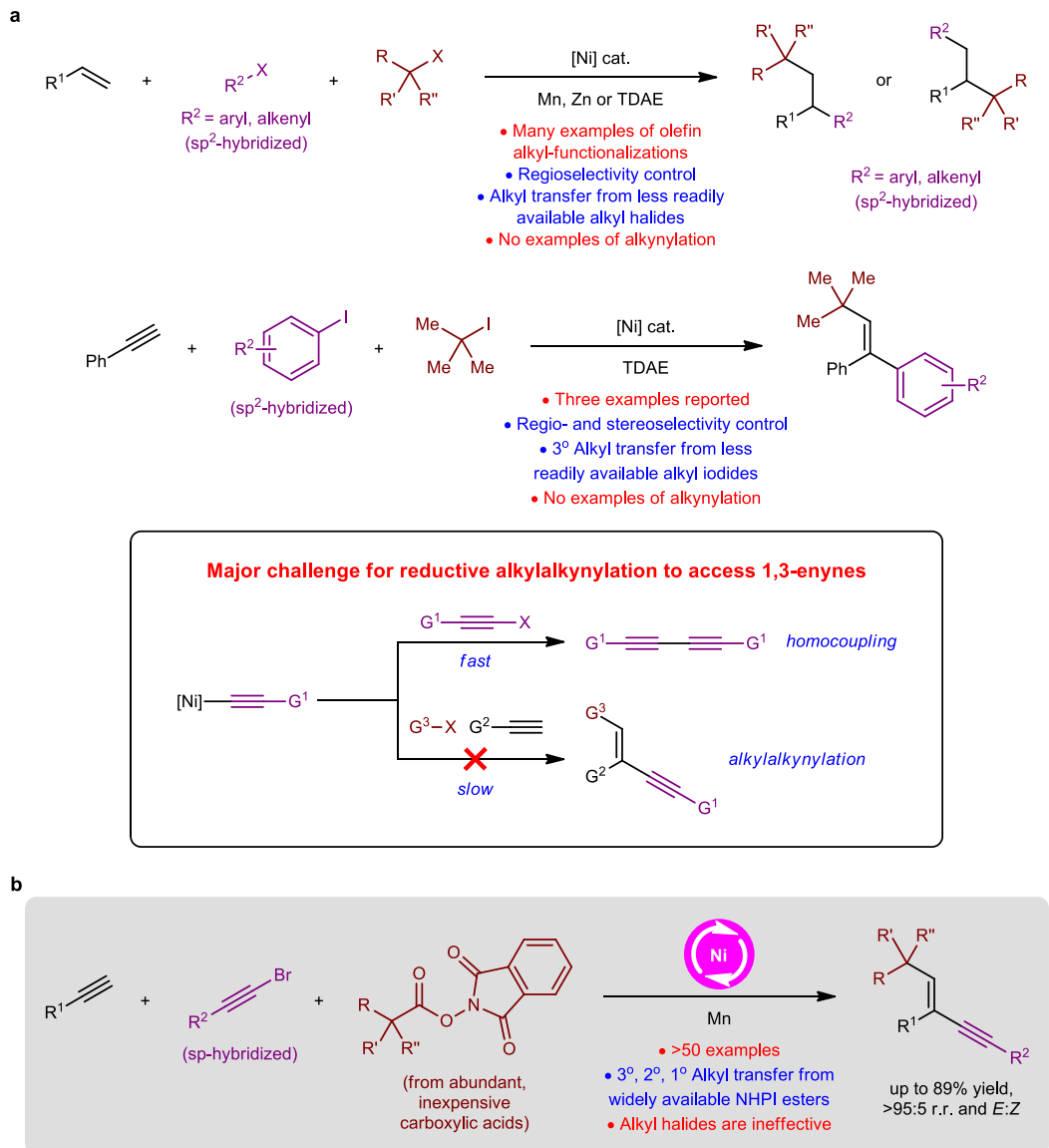

Fig. 1 | The significance of developing site- and stereoselective reductive alkyne alkylalkynylation. a, State-of-the-art advances in multicomponent reductive alkyl-functionalizations of unsaturated $\mathrm{C}-\mathrm{C}$ bonds. Examples of reductive additions to alkynes with sp-hybridized electrophiles are yet to be reported, presumably due to the difficulties of overcoming rapid homocoupling of the reactive alkynyl halide. b, Ni-catalyzed reductive alkylalkynylation of alkynes using NHPI esters and haloalkynes offers a convenient strategy to assemble stereodefined 1,3-enynes in one step by exploiting widely available redox-active esters as efficient alkyl group donors. R, functional group; $\mathrm{X}$, halide; cat., catalyst; NHPI, N-hydroxyphthalimide; TDAE, tetrakis(dimethylamino)ethylene. 
A growing class of three-component dicarbofunctionalization reactions pertain to the regioselective addition of carbogenic groups, derived from stable electrophilic organohalides (vs. the more sensitive organometallic reagents $\left.{ }^{55-56}\right)$, across $C-C \pi$ bonds in the presence of a mild reducing agent. ${ }^{57-62}$ To date, most reductive alkyl-functionalization processes involve alkyl-aryl or alkyl-alkenyl additions to olefins using iodo- or bromoalkanes as the alkyl group donor (Fig. 1a). In contrast, the corresponding transformations with alkynes are severely under-developed and restricted to alkyl-arylations using organoiodide reagents. ${ }^{57}$ One longstanding challenge that arises from three-component reductive alkynylation processes is the high propensity of the haloalkyne electrophile to undergo facile homocoupling in the presence of a Ni-based complex, inadvertently suppressing the desired alkylalkynylation pathway (Fig. 1a, inset; see Fig. 4 for further discussion). Notwithstanding these limitations, we reasoned that the union of an alkyne, an alkynyl halide and a redox-active NHPI ester can be achieved using a Ni-based catalyst under appropriate reductive conditions to give diverse 1,3-enynes with simultaneous control of site and stereoselectivity (Fig. 1b).

Our motivation to pursue this approach is twofold: (1) The greater variety of $N$ (acyloxy)phthalimides accessible from aliphatic carboxylic acids (vs. alkyl halides) means that diverse aliphatic units (tertiary, secondary, primary) can be incorporated; (2) The ability of NHPI esters to promote challenging alkylalkynylations in which alkyl halides fail to deliver, by minimizing rampant undesired pathways arising from homocoupling ${ }^{63-64}$ and cross-coupling ${ }^{14,16}$ of the alkynyl halide and NHPI ester reactants, as well as alkyne cyclotrimerization ${ }^{65}$ (see Fig. 4 for further discussion). Herein, we disclose the first reductive protocol that accomplishes selective alkyne alkylalkynylation using NHPI esters as efficient aliphatic group donors.

\section{Results}




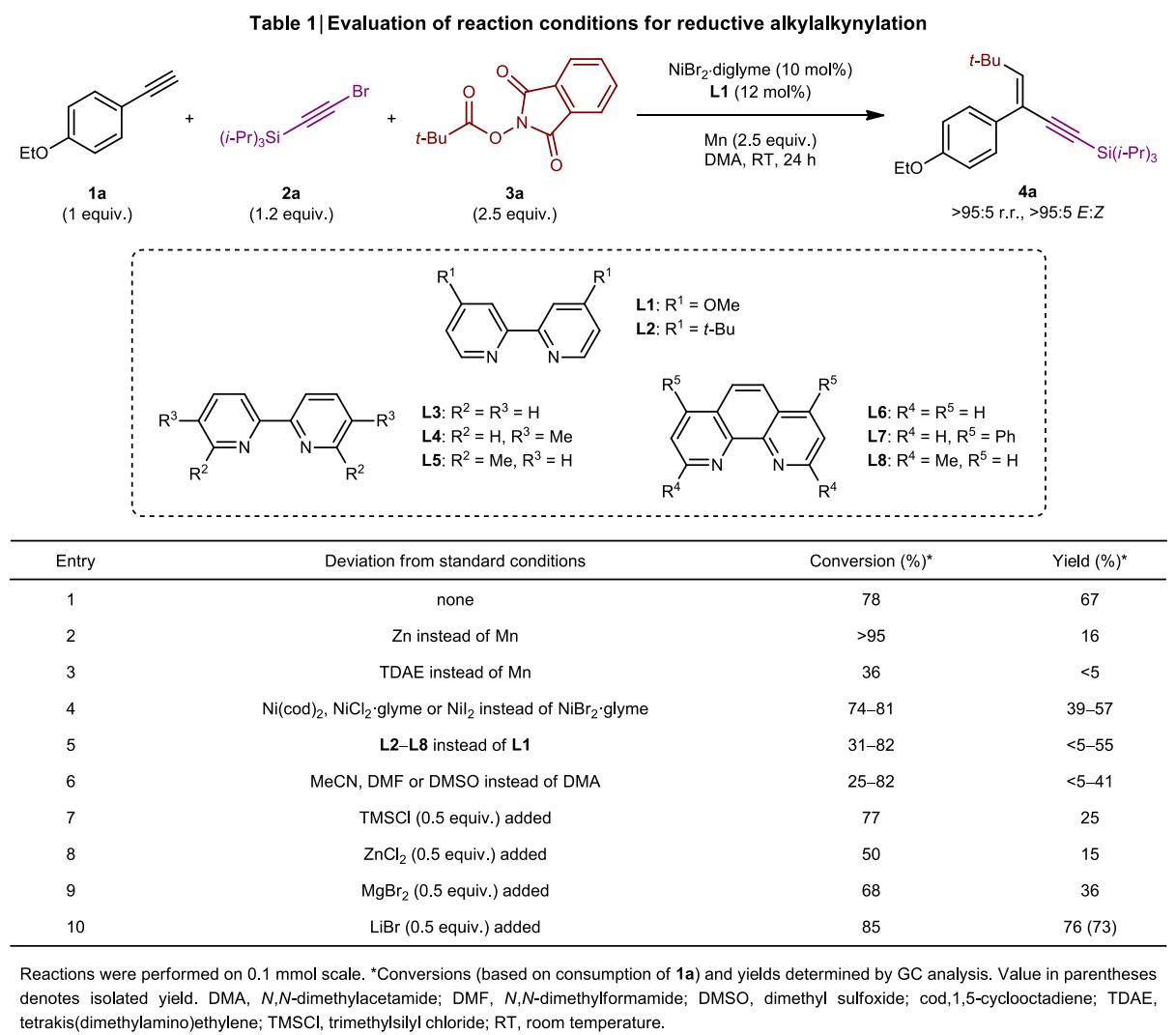

Reaction optimization. Examination of conditions for the reaction of 1a (1 equiv.), 2a (1.2 equiv.) and $3 a$ ( 2.5 equiv.) showed that the desired 1,3-enyne product 4 a could be obtained in $67 \%$ GC yield ( $>95 \%$ regio- and $E$ selectivity) in the presence of $10 \mathrm{~mol} \%$ of the $\mathrm{Ni}$-based complex derived from $\mathrm{NiBr}_{2}$. diglyme and $\mathrm{L1}, \mathrm{Mn}$ (2.5 equiv.) and DMA as solvent under ambient conditions (Table 1 , entry 1). Switching the reducing agent to $\mathrm{Zn}$ or tetrakis(dimethylamino)ethylene (TDAE) led to poor yields of $\mathbf{4 a}$ with excessive by-product formation from $1 \mathbf{a}$ cyclotrimerization ${ }^{65}$ and $2 \mathrm{a}$ homocoupling ${ }^{63-64}$ (Table 1, entries 2 and 3). Other Ni-based complexes were less effective in promoting alkylalkynylation (Table 1, entry 4), while less electron-rich bipyridine and phenanthroline ligands $\mathbf{L 2}-\mathbf{L} \mathbf{8}$ afforded $\mathbf{4 a}$ in unsatisfactory yields (Table 1, entry 5). Changing DMA to other polar solvents also did not improve results (Table 1, entry 6).

In order to enhance the catalytic efficiency and/or suppress the undesired formation of diyne by-products, ${ }^{63-64}$ various additives were experimented as detailed in Table 1, entries 7-10. Addition of TMSCl (known to activate the $\mathrm{Mn}(0)$ surface ${ }^{66}$ ) to the reaction system was somewhat detrimental (Table 1, entry 7), whereas $\mathrm{ZnCl}_{2}$ or $\mathrm{MgBr}_{2}$ additives ${ }^{67}$ also reduced the yield of 4 a (Table 1, entries 8 and 9). Considering the previously reported role of lithium salts in minimizing 
diyne formation, ${ }^{14}$ we found that the use of $\mathrm{LiBr}$ ( 0.5 equiv.) indeed improved results, affording $4 a$ in $76 \%$ yield ( $73 \%$ isolated; Table 1 , entry 10$)$.

Substrate scope. To examine the generality of the established conditions, we tested a range of electronically and sterically diverse aryl- and heteroaryl-substituted alkynes, and the desired products $\mathbf{4 b}$-aa were isolated in $40-81 \%$ yield as single regio- and $E$ isomers (Fig. 2). Both electron-rich and electron-deficient arenes are tolerated, including those that contain a Lewis basic aniline (4g), Brønsted acidic NHBoc (4h) and electrophilic aldehyde (4k). Synthesis of $\mathbf{4 j}(<5 \%$ hydrodebromination side products) that is functionalized with a bromoaryl substituent highlights the transformation's remarkable chemoselectivity. As demonstrated by the preparation of $\mathbf{4 b}$, the transformations may be performed on larger scale $(3 \mathrm{mmol}$ ) without appreciable diminution in efficiency.

Products that bear heterocyclic units (4q and $\mathbf{4 r}$ ), as well as those derived from complex bioactive compounds $(\mathbf{4} \mathbf{u}-\mathbf{w})$ could be generated. By using a D-substituted alkyne, tetrasubstituted deuterium-labelled olefins such as $\mathbf{4 x}$, which otherwise might be difficult to prepare by other means, could be secured through the present protocol. However, internal alkynes were resistant to alkylalkynylation (cf. $5 ;<5 \%$ conv. to product). Aliphatic alkynes were also found to be ineffective substrates under the standard conditions. Besides silyl-substituted bromoalkynes, aryl- and alkyl-functionalized alkynyl bromides also underwent efficient reaction to deliver the expected 1,3-enynes $4 \mathbf{y}$-aa in $46-53 \%$ yield and $94-95 \% E$ selectivity. 

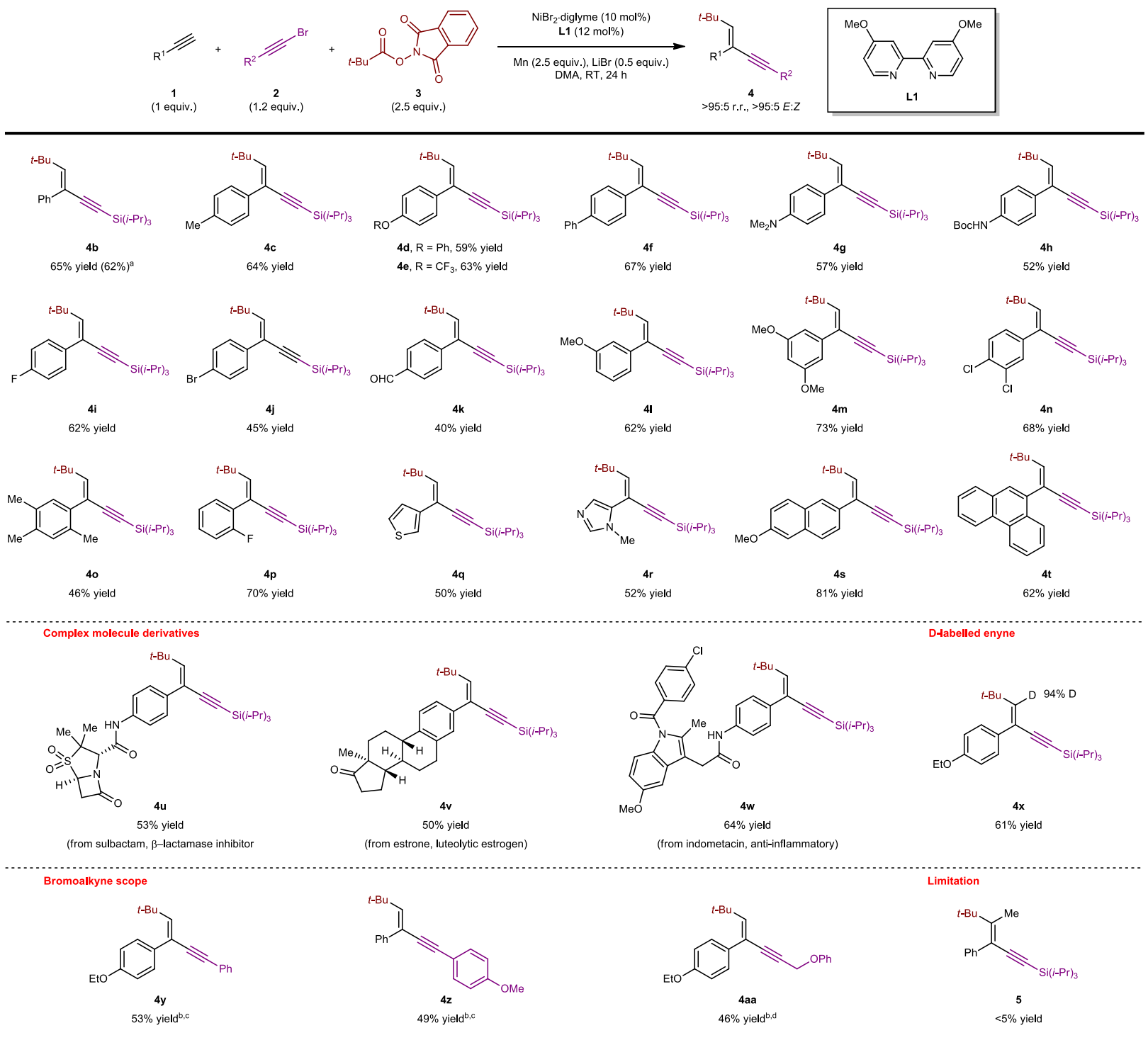

Fig. 2 The scope of alkynes and alkynyl bromides. Regioisomeric ratios (r.r.) and $E: Z$ ratios were determined by GC and ${ }^{1} \mathrm{H}$ NMR

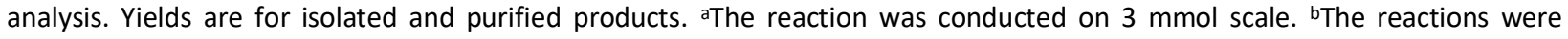
conducted with $\mathrm{LiBr}$ (1 equiv.) and DMSO as solvent. ${ }^{\mathrm{T} T h e}$ products were generated in 95:5 E:Z ratio. dThe product was generated in 94:6 E:Z ratio. $\mathrm{R}$, functional group; $\mathrm{DMA}, \mathrm{N}, \mathrm{N}$-dimethylacetamide; $\mathrm{RT}$, room temperature; Boc, tert-butoxycarbonyl.

A wide assortment of aliphatic NHPI esters served as effective reagents for alkylalkynylation (Fig. 3). These include tertiary alkyl $N$-(acyloxy)phthalimides (affording 4ab-ar with quaternary carbon centers), secondary alkyl $\mathrm{N}$-(acyloxy)phthalimides (affording 4au and 4av with tertiary carbon centers) as well as primary alkyl $N$-(acyloxy)phthalimides (4aw and 4ax). To facilitate secondary and primary alkyl additions, an additional $10 \mathrm{~mol} \%$ of CuTC was added as co-catalyst to improve yields, possibly by stabilization of the corresponding alkyl radicals generated. ${ }^{68}$ The diversity of aliphatic groups which can be installed (such as oxetane 4af, pyrans 4ak and 4al, 
piperidine 4an and acid-labile acetal 4am) compares favorably with previous methods that employed less readily available haloalkanes. ${ }^{57,60,69}$
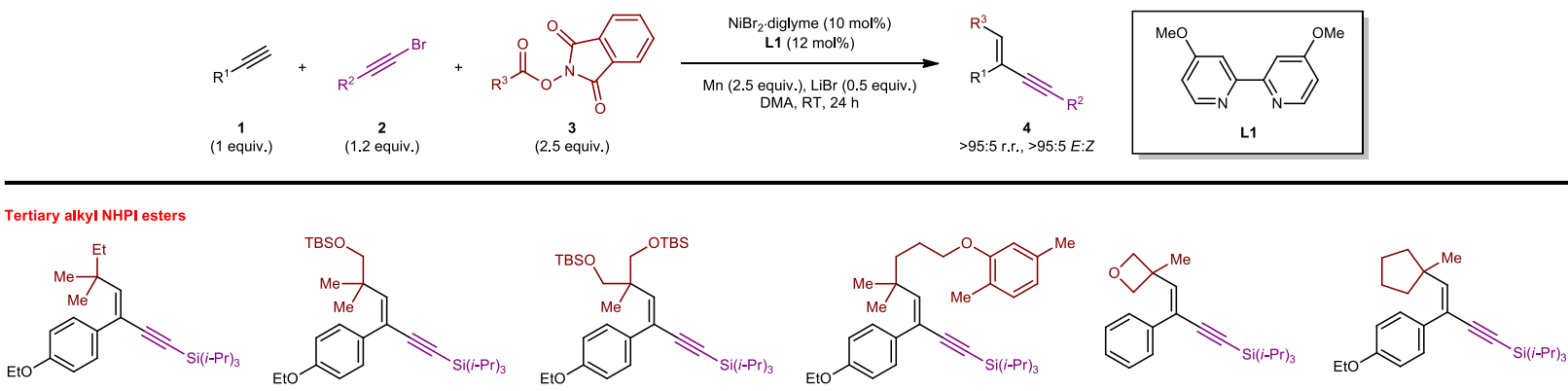

4ab
$61 \%$ yield

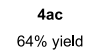

4 ad
$70 \%$ yield

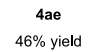

4af
$30 \%$ yield $^{\mathrm{a}}$

4 ag
$55 \%$ yield
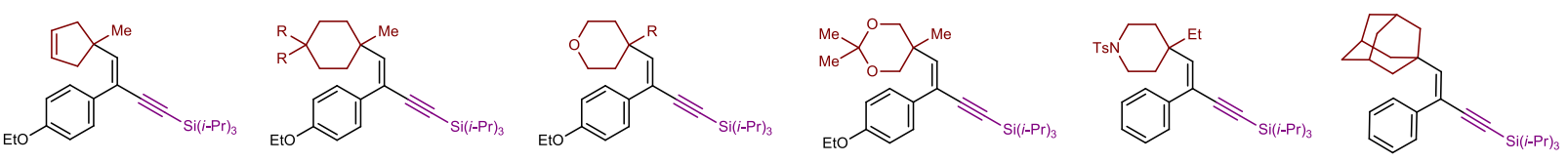

4ah
$47 \%$ yield

4ai, $R=H, 74 \%$ yield
4aj, $R=F, 89 \%$ yield

\section{4ak, $R=$ Me, $76 \%$ yield}

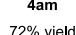

4an

$50 \%$ yield

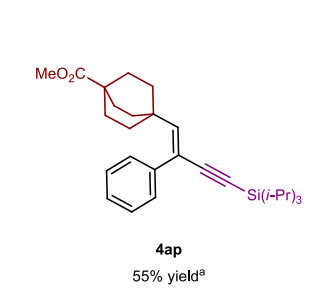

Complex molecule derivatives

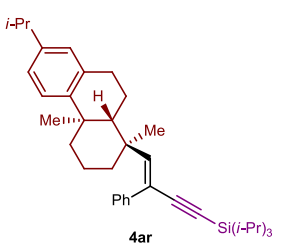

$58 \%$ yielda, $10: 1$ d.r. (from dehydroabietic acid, diterpenoid)

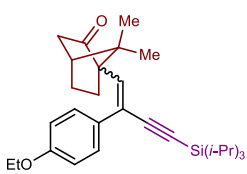

4aq

$41 \%$ yield, $1: 1$ d.r. (from $(S)-(+)$-ketopinic acid)

Secondary and primary alkyl NHPI esters
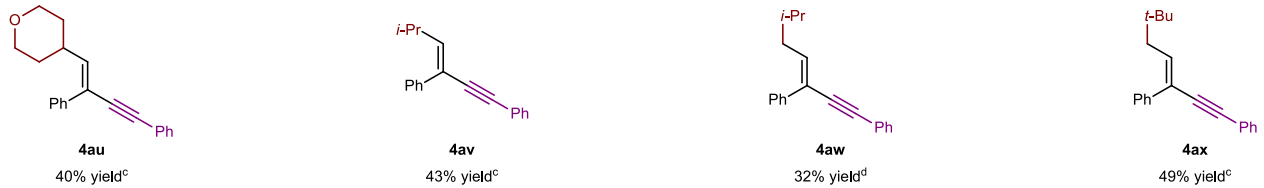

Fig. 3 | The scope of redox-active esters. Regioisomeric ratios (r.r.), diastereomeric ratios (d.r.) and $E: Z$ ratios were determined by GC and ${ }^{1} \mathrm{H}$ NMR analysis. Yields are for isolated and purified products. ${ }^{a}$ The reactions were conducted with $\mathbf{1}$ (3 equiv.) and $\mathbf{2}$

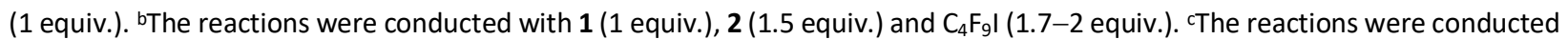
with 1 (3 equiv.), $\mathbf{2}$ ( 1 equiv.) and LiBr (1 equiv.) with $\mathbf{L} \mathbf{4}(12 \mathrm{~mol} \%)$ as ligand and CuTC (10 mol \%) as co-catalyst. dThe reactions were conducted with 1 ( 3 equiv.), $\mathbf{2}$ (1 equiv.) and LiBr (1 equiv.) with L1 (12 mol\%) as ligand and CuTC (10 mol \%) as co-catalyst. R, functional group; DMA, $N, N$-dimethylacetamide; RT, room temperature; TBS, tert-butyldimethylsilyl; Ts, $p$-toluenesulfonyl; TC, thiophene-2-carboxylate.

Structurally sophisticated alkyl additions could be implemented as exemplified by the products 4aq (from ketopinic acid) and 4ar (from dehydroabietic acid). To incorporate fluoroalkyl units, due to the difficulty of fluoroalkyl NHPI esters to generate the requisite fluorinated radical species, ${ }^{70}$ we turned to perfluoroalkyl iodide to deliver alkylalkynylation of both aryl- and alkylsubstituted alkynes. In the event, the F-containing 1,3-enynes 4as and 4at were successfully isolated in $53 \%$ and $36 \%$ yields, respectively. 
Mechanistic studies. As shown in Fig. 4, studies were carried out to elucidate the mechanism of the reductive alkyne alkylalkynylation process.

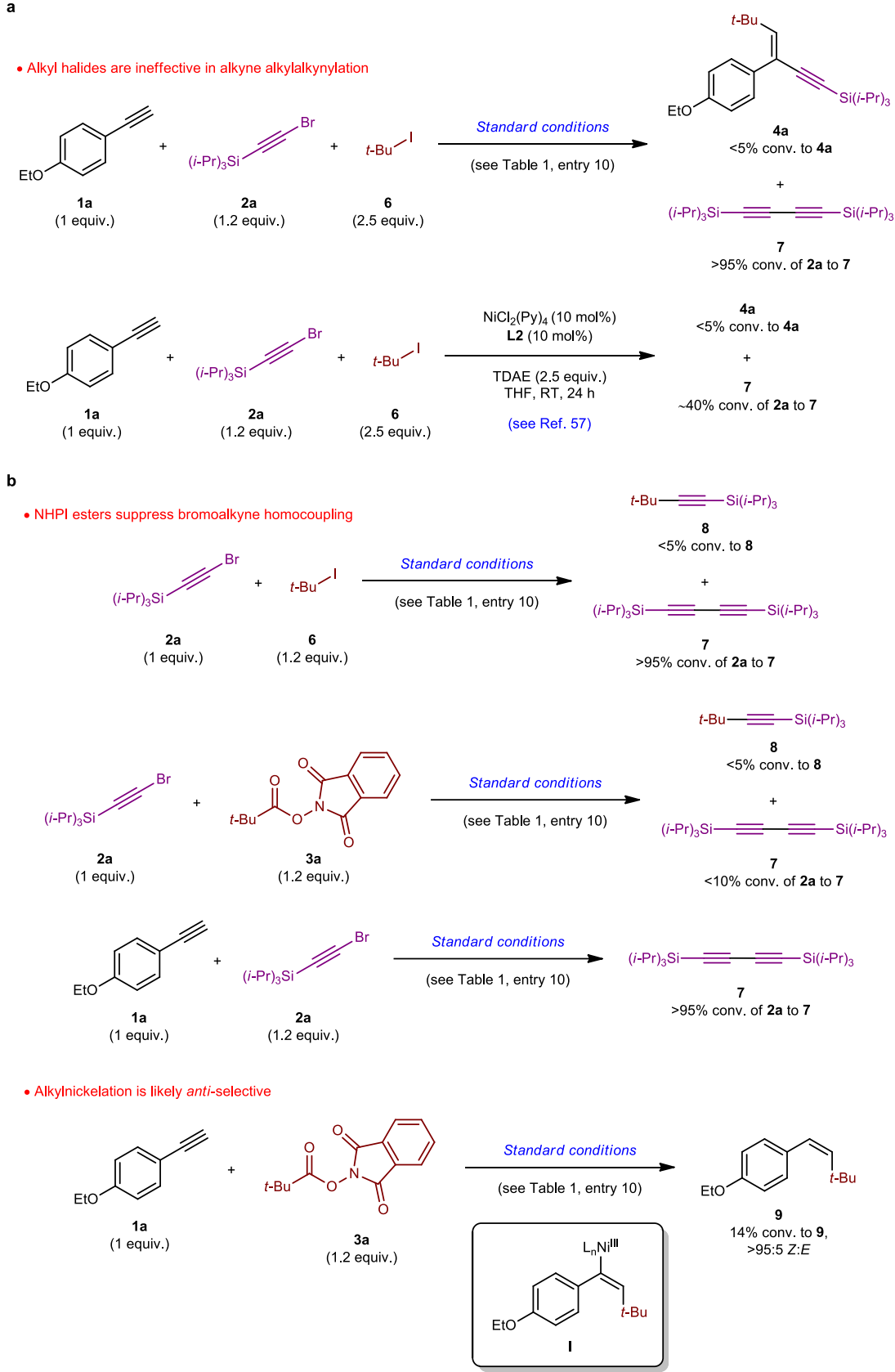

Fig. 4 | Mechanistic investigations. a, Unsuccessful alkylalkynylation attempts using iodoalkane as alkyl group donor. b, Twocomponent control experiments. R, functional group; L, ligand; THF, tetrahydrofuran; Py, pyridine; TDAE, tetrakis(dimethylamino)ethylene; RT, room temperature.

Remarkably, control experiments showed that when NHPI ester 3a was replaced by the corresponding 2 -iodo-2-methylpropane 6 , there was $<5 \%$ conv. to the 1,3 -enyne product 4 a. Instead, the alkyne 1a was fully consumed in cyclotrimerization ${ }^{65}$ to form arene side products, 
and homocoupling of bromoalkyne $\mathbf{2 a}$ to give diyne $\mathbf{7}$ was detected (Fig. 4a). Repeating the reaction under previously established reductive dicarbofunctionalization conditions ${ }^{57}$ also did not yield $4 a$ ( $<5 \%$ conv. of $1 a, \sim 40 \%$ conv. of $2 a$ to 7 ). These observations not only highlight the importance of the redox-active ester component as an effective alkyl donor in these multicomponent reactions, but also provide hints that the alkynyl bromide was probably much more reactive (vs. the alkyl iodide), inadvertently suppressing the desired alkylalkynylation pathway and causing homocoupling of the bromoalkyne to predominate.

Additional control experiments shed further light on the reaction (Fig. 4b). Under standard conditions, the reaction between bromoalkyne $\mathbf{2 a}$ and $\mathbf{6}$ led to full conversion of $\mathbf{2 a}$ to diyne $\mathbf{7}$ ( $<5 \%$ cross-coupling to $\mathbf{8}$ detected). When alkyne $\mathbf{1 a}$ was treated with $\mathbf{2 a}$ under the same conditions, $>95 \%$ conv. to 7 was also detected and 1a underwent undesired cyclotrimerization. In contrast, replacing the iodoalkane 6 with NHPI ester 3a only afforded trace amounts of 7 ( $<5 \%$ cross-coupling to $\mathbf{8}$ ) and $\mathbf{3 a}$ was fully consumed (presumably by decomposition under the reductive conditions ${ }^{71}$ ). These observations imply that the presence of $\mathbf{3 a}$ somehow inhibited $\mathbf{2 a}$ homocoupling by preferentially engaging with an in situ-generated organonickel species, albeit no productive reaction could occur if alkyne 1a was absent to trap the $t$-Bu radical formed (see Fig. 5). Notably, subjecting $1 \mathbf{1 a}$ to $\mathbf{3 a}$ under the established conditions selectively furnished $Z$ alkene 9 in 14\% GC yield, leading us to deduce that the $\mathrm{C}-\mathrm{C}(\mathrm{t}-\mathrm{Bu})$ bond and the adjacent $\mathrm{C}-\mathrm{Ni}$ bond are generated in an anti configuration (presumably to minimize steric repulsions) within the alkenylnickel intermediate I (see Fig. 5). In the absence of the alkynyl bromide, 9 might be formed by adventitious protodemetallation of I with residual moisture.

Based on our investigations and related studies, ${ }^{14,60,64}$ a tentative mechanism is proposed in Fig. 5. Starting from an in situ-generated $\mathrm{Ni}(0)$ species $\mathbf{i}$ (e.g. from reduction of the Ni(II) precatalyst ${ }^{14,64}$, oxidative addition with bromoalkyne 2 followed by single-electron reduction in the presene of $\mathrm{Mn}$ gives rise to an alkynylnickel(I) species iii. At this stage, a second molecule of 2 could potentially react with iii to give dialkynylnickel(III) vii that subsequently reductively eliminates to afford the undesired diyne side product 7. However, if a NHPI ester $\mathbf{3}$ is present in the system, the reaction trajectory could be altered as $\mathbf{3}$ chemoselectively engages with iii, through a single-electron transfer (SET) decarboxylative pathway, ${ }^{14}$ to furnish alkynylnickel(II) complex iv with concomitant ejection of $\mathrm{CO}_{2}$, phthalimide anion and an alkyl radical species. Facile capture of the alkyl radical by alkyne 1 generates an alkenyl radical that recombines with iv to form E-alkenylnickel(III) complex v. The ensuing reductive elimination then generates $\mathrm{Ni}(\mathrm{I})$ 
phthalimide vi and releases the desired 1,3-enyne 4. Following another single-electron reduction by $\mathrm{Mn}, \mathbf{i}$ is regenerated to turn over the catalytic cycle. On the other hand, a less reactive alkyl halide (vs. alkyl NHPI ester) is not capable of efficiently intercepting alkynylnickel(I) complex iii, consequently allowing other side reactions such as homocoupling $\mathbf{2}$ to become competitive in the system.

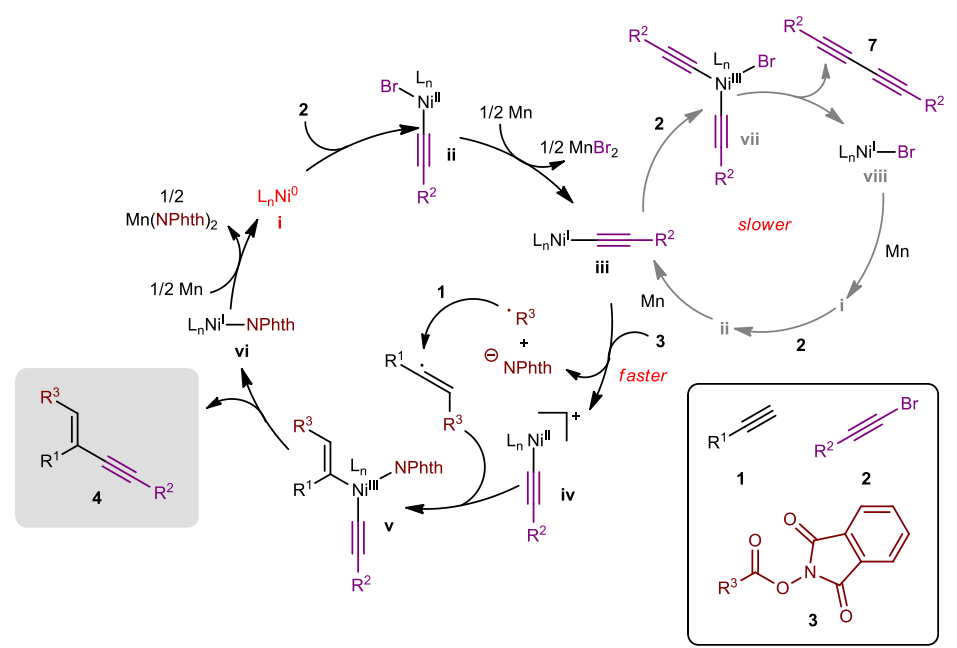

Fig. 5 | Proposed catalytic mechanism for reductive alkylalkynylation. Unlike a haloalkane, NHPI ester $\mathbf{3}$ is capable of intercepting the putative alkynylnickel intermediate iii to promote alkylalkynylation and suppress adventitious homocoupling of $\mathbf{2}$ to diyne $\mathbf{7}$. $\mathrm{R}$, functional group; L, ligand; Phth, phthaloyl.

Synthetic transformations. Using redox-active esters 10 tethered to a terminal olefin, we postulated that a cascade pathway ${ }^{72}$ commencing from alkyl radical addition to the alkyne followed by an intramolecular 5-exo-trig cyclization with the $\mathrm{C}=\mathrm{C}$ bond to give a second alkyl radical species III before reassociation with the $\mathrm{Ni}$ complex for subsequent alkynylation could occur (Fig. 6a). This would give rise to complex 1,5-enynes 11 bearing a trisubstituted cyclopentene nucleus and an alkyne appendage. Gratifyingly, the Ni-catalyzed cascade processes proceeded smoothly to generate the desired products $11 \mathbf{a}-\mathbf{e}$ in up to $85 \%$ yield, further demonstrating the versatility of the alkylalkynylation regime by taking advantage of radical-based reactivity modes to construct complex molecules. 
a
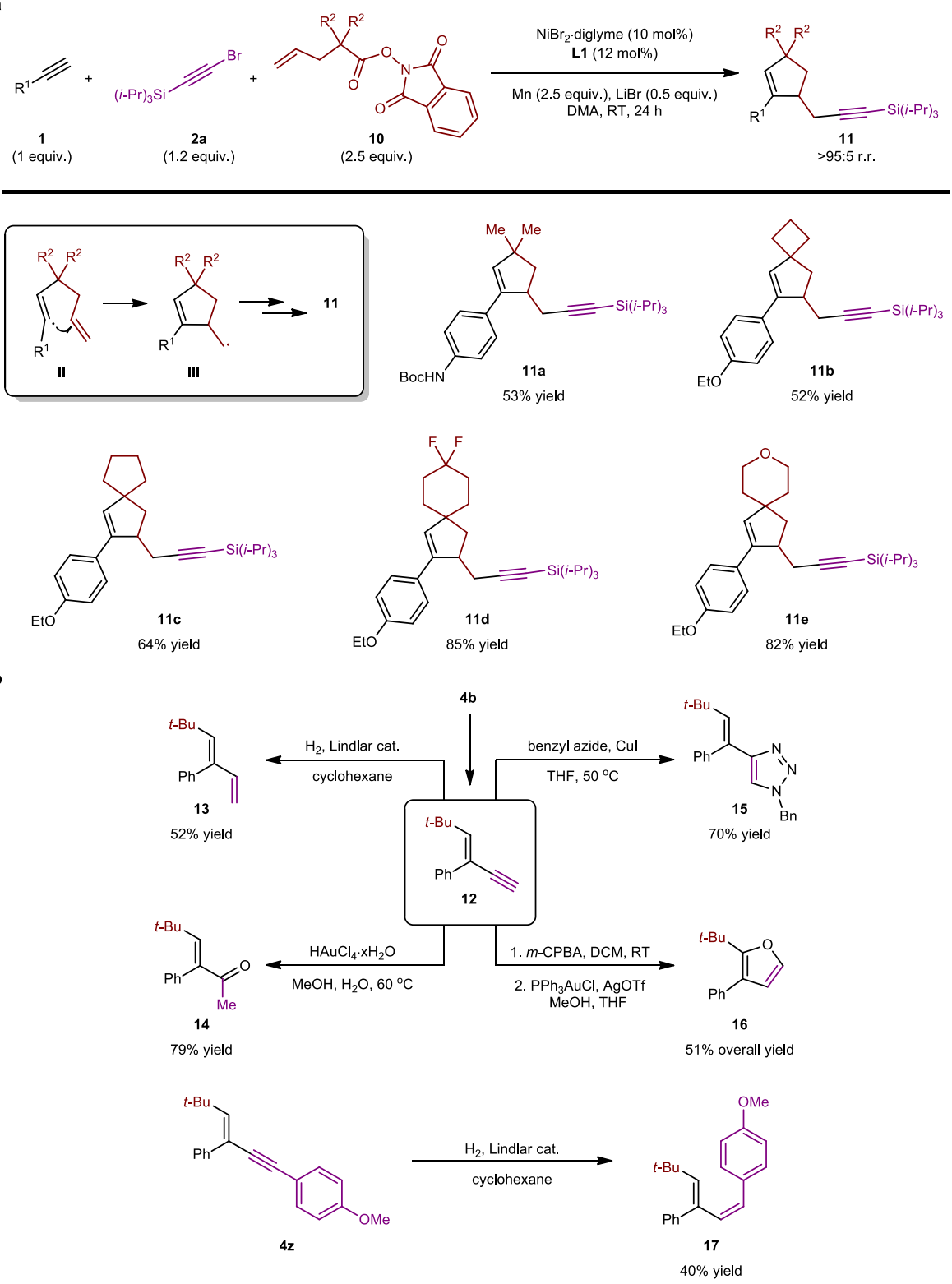

Fig. 6 | Application to cascade processes and further derivatization. a, Cascade radical addition/cyclization/alkynylation to furnish 1,5-enynes. b, Chemical transformations of stereodefined 1,3-enynes to synthetically valuable building blocks. R, functional group; DMA, N,N-dimethylacetamide; DCM, dichloromethane; THF, tetrahydrofuran; RT, room temperature; cat., catalyst; Bn, benzyl; Boc, tert-butoxycarbonyl; Tf, trifluoromethanesulfonyl; m-CPBA; meta-chloroperoxybenzoic acid.

Utility of the 1,3-enyne products is showcased through a series of synthetic manipulations involving both the olefin and alkyne motifs towards the preparation of diverse molecular structures (Fig. 6b). Using the desilylated derivative $\mathbf{1 2}$ from $\mathbf{4 b},{ }^{73}$ facile transformation of the terminal alkyne moiety to a spectrum of different products can be effected by partial hydrogenation to the 1,3-diene 13 in 52\% yield, ${ }^{74}$ Au-catalyzed hydration to ketone 14 in $79 \%$ yield, ${ }^{75}$ and Cu-catalyzed azide-alkyne cycloaddition to $1,2,3$-triazole 15 in $70 \%$ yield. ${ }^{76}$ In another 
instance, chemoselective epoxidation of the trisubstituted olefin followed by Au-catalyzed cycloisomerization ${ }^{77}$ afforded the disubstituted furan derivative 16 in 51\% overall yield. On the other hand, partial cis-selective hydrogenation of the internal alkyne in $\mathbf{4 z}$ generated sterically congested 1,3-diene $\mathbf{1 7}$ in $40 \%$ yield as a single $Z$ isomer.

To conclude, we have demonstrated that a single $\mathrm{Ni}$-based catalyst is capable of mediating regio- and stereoselective alkyl-alkynyl additions to alkynes to deliver valuable 1,3-enyne products. Access to 1,5-enynes was achieved through a radical-based cascade transformation, and our investigations shed light on the superior performance of redox-active esters in overcoming undesired haloalkyne homocoupling by competitively intercepting a putative alkynyInickel intermediate. In situations where two electrophilic halides proved to be ineffective, the synergistic combination of a redox-active ester and an organohalide may provide viable solutions to address other longstanding challenges in dicarbofunctionalizations that employ multiple electrophiles.

\section{Data availability}

All data are available from the corresponding authors upon reasonable request.

\section{References}

1. Straathof, A. J. Transformation of biomass into commodity chemicals using enzymes or cells. Chem. Rev. 114, 1871-1908 (2014).

2. Patra, T. \& Maiti, D. Decarboxylation as the key step in C-C bond-forming reactions. Chem. Eur. J. 23, 73827401 (2017).

3. Xuan, J., Zhang, Z. G. \& Xiao, W. J. Visible-light-induced decarboxylative functionalization of carboxylic acids and their derivatives. Angew. Chem. Int. Ed. 54, 15632-15641 (2015).

4. Kautzky, J. A., Wang, T., Evans, R. W. \& MacMillan, D. W. C. Decarboxylative trifluoromethylation of aliphatic carboxylic acids. J. Am. Chem. Soc. 140, 6522-6526 (2018).

5. Till, N. A., Smith, R. T. \& MacMillan, D. W. C. Decarboxylative hydroalkylation of alkynes. J. Am. Chem. Soc. 140, 5701-5705 (2018).

6. Rahman, M. et al. Recent advances on diverse decarboxylative reactions of amino acids. Adv. Synth. Catal. 361, 2161-2214 (2019).

7. McMurray, L., McGuire, T. M. \& Howells, R. L. Recent advances in photocatalytic decarboxylative coupling reactions in medicinal chemistry. Synthesis 52, 1719-1737 (2020).

8. Mega, R. S., Duong, V. K., Noble, A. \& Aggarwal, V. K. Decarboxylative conjunctive cross-coupling of vinyl boronic esters using metallaphotoredox catalysis. Angew. Chem. Int. Ed. 59, 4375-4379 (2020).

9. Wang, H. et al. Engaging $\alpha$-fluorocarboxylic acids directly in decarboxylative $\mathrm{C}-\mathrm{C}$ bond formation. ACS Catal. 10, 4451-4459 (2020).

10. Yue, H., Zhu, C., Kancherla, R., Liu, F. \& Rueping, M. Regioselective hydroalkylation and arylalkylation of alkynes by photoredox/nickel dual catalysis: Application and mechanism. Angew. Chem. Int. Ed. 59, 57385746 (2020).

11. Cornella, J. et al. Practical Ni-catalyzed aryl-alkyl cross-coupling of secondary redox-active esters. J. Am. Chem. Soc. 138, 2174-2177 (2016). 
12. Qin, T. et al. A general alkyl-alkyl cross-coupling enabled by redox-active esters and alkylzinc reagents. Science 352, 801-805 (2016).

13. Edwards, J. T. et al. Decarboxylative alkenylation. Nature 545, 213-218 (2017).

14. Huang, L., Olivares, A. M. \& Weix, D. J. Reductive decarboxylative alkynylation of $N$-hydroxyphthalimide esters with bromoalkynes. Angew. Chem. Int. Ed. 56, 11901-11905 (2017).

15. Sandfort, F., O'Neill, M. J., Cornella, J., Wimmer, L. \& Baran, P. S. Alkyl-(hetero)aryl bond formation via decarboxylative cross-coupling: A systematic analysis. Angew. Chem. Int. Ed. 56, 3319-3323 (2017).

16. Smith, J. M. et al. Decarboxylative alkynylation. Angew. Chem. Int. Ed. 56, 11906-11910 (2017).

17. Murarka, S. N-(acyloxy)phthalimides as redox-active esters in cross-coupling reactions. Adv. Synth. Catal. 360, 1735-1753 (2018).

18. Ni, S. et al. A general amino acid synthesis enabled by innate radical cross-coupling. Angew. Chem. Int. Ed. 57, 14560-14565 (2018).

19. Chen, T.-G. et al. Quaternary centers by nickel-catalyzed cross-coupling of tertiary carboxylic acids and (hetero)aryl zinc reagents. Angew. Chem. Int. Ed. 58, 2454-2458 (2019).

20. Ishii, T., Kakeno, Y., Nagao, K. \& Ohmiya, H. N-heterocyclic carbene-catalyzed decarboxylative alkylation of aldehydes. J. Am. Chem. Soc. 141, 3854-3858 (2019).

21. Ni, S. et al. A radical approach to anionic chemistry: Synthesis of ketones, alcohols, and amines. J. Am. Chem. Soc. 141, 6726-6739 (2019).

22. Wang, J., Cary, B. P., Beyer, P. D., Gellman, S. H. \& Weix, D. J. Ketones from nickel-catalyzed decarboxylative, non-symmetric cross-electrophile coupling of carboxylic acid esters. Angew. Chem. Int. Ed. 58, 12081-12085 (2019).

23. Kakeno, Y., Kusakabe, M., Nagao, K. \& Ohmiya, H. Direct synthesis of dialkyl ketones from aliphatic aldehydes through radical $N$-heterocyclic carbene catalysis. ACS Catal. 10, 8524-8529 (2020).

24. Johnston, C. P., Smith, R. T., Allmendinger, S. \& MacMillan, D. W. Metallaphotoredox-catalysed $s p^{3}-s p^{3}$ cross-coupling of carboxylic acids with alkyl halides. Nature 536, 322-325 (2016).

25. Dai, G.-L., Lai, S.-Z., Luo, Z. \& Tang, Z.-Y. Selective syntheses of Z-alkenes via photocatalyzed decarboxylative coupling of $N$-hydroxyphthalimide esters with terminal arylalkynes. Org. Lett. 21, 2269-2272 (2019).

26. Chu, L., Ohta, C., Zuo, Z. \& MacMillan, D. W. Carboxylic acids as a traceless activation group for conjugate additions: A three-step synthesis of (+/-)-pregabalin. J. Am. Chem. Soc. 136, 10886-10889 (2014).

27. Jian, W., Ge, L., Jiao, Y., Qian, B. \& Bao, H. Iron-catalyzed decarboxylative alkyl etherification of vinylarenes with aliphatic acids as the alkyl source. Angew. Chem., Int. Ed. 56, 3650-3654 (2017).

28. Qin, T. et al. Nickel-catalyzed barton decarboxylation and giese reactions: A practical take on classic transforms. Angew. Chem. Int. Ed. 56, 260-265 (2017).

29. Tlahuext-Aca, A., Garza-Sanchez, R. A. \& Glorius, F. Multicomponent oxyalkylation of styrenes enabled by hydrogen-bond-assisted photoinduced electron transfer. Angew. Chem. Int. Ed. 56, 3708-3711 (2017).

30. Bloom, S. et al. Decarboxylative alkylation for site-selective bioconjugation of native proteins via oxidation potentials. Nat. Chem. 10, 205-211 (2018).

31. Tlahuext-Aca, A., Garza-Sanchez, R. A., Schafer, M. \& Glorius, F. Visible-light-mediated synthesis of ketones by the oxidative alkylation of styrenes. Org. Lett. 20, 1546-1549 (2018).

32. Wang, G. Z., Shang, R. \& Fu, Y. Irradiation-induced palladium-catalyzed decarboxylative heck reaction of aliphatic $N$-(acyloxy)phthalimides at room temperature. Org. Lett. 20, 888-891 (2018).

33. Wang, J. et al. Kinetically guided radical-based synthesis of $C\left(s p^{3}\right)-C\left(s p^{3}\right)$ linkages on DNA. Proc. Natl. Acad. Sci. USA 115, E6404-E6410 (2018).

34. Guo, J. Y. et al. Photoredox-catalyzed stereoselective alkylation of enamides with $N$-hydroxyphthalimide esters via decarboxylative cross-coupling reactions. Chem. Sci. 10, 8792-8798 (2019).

35. Ishii, T., Ota, K., Nagao, K. \& Ohmiya, H. N-heterocyclic carbene-catalyzed radical relay enabling vicinal alkylacylation of alkenes. J. Am. Chem. Soc. 141, 14073-14077 (2019). 
36. Dang, H. T. et al. Acridine photocatalysis: Insights into the mechanism and development of a dual-catalytic direct decarboxylative conjugate addition. ACS Catal. 10, 11448-11457 (2020).

37. Huang, H.-M. et al. Catalytic radical generation of $\pi$-allylpalladium complexes. Nat. Catal. 3, 393-400 (2020).

38. Zein, N., Sinha, A., McGahren, W. \& Ellestad, G. Calicheamicin gamma $\gamma_{1}{ }^{1}$ : An antitumor antibiotic that cleaves double-stranded DNA site specifically. Science 240, 1198-1201 (1988).

39. Nussbaumer, P., Leitner, I., Mraz, K. \& Stuetz, A. Synthesis and structure-activity relationships of side-chainsubstituted analogs of the allylamine antimycotic terbinafine lacking the central amino function. J. Med. Chem. 38, 1831-1836 (1995).

40. Myers, A. G. et al. Enantioselective synthesis of neocarzinostatin chromophore aglycon. J. Am. Chem. Soc. 118, 10006-10007 (1996).

41. Hussain, H., Green, I. R., Krohn, K. \& Ahmed, I. Advances in the total synthesis of biologically important callipeltosides: A review. Nat. Prod. Rep. 30, 640-693 (2013).

42. Ma, X., Banwell, M. G. \& Willis, A. C. Chemoenzymatic total synthesis of the phytotoxic geranylcyclohexentriol (-)-phomentrioloxin. J. Nat. Prod. 76, 1514-1518 (2013).

43. Cornelissen, L., Lefrancq, M. \& Riant, O. Copper-catalyzed cross-coupling of vinylsiloxanes with bromoalkynes: Synthesis of enynes. Org. Lett. 16, 3024-3027 (2014).

44. Che, C., Zheng, H. \& Zhu, G. Copper-catalyzed trans-carbohalogenation of terminal alkynes with functionalized tertiary alkyl halides. Org. Lett. 17, 1617-1620 (2015).

45. Cheung, C. W. \& Hu, X. Stereoselective synthesis of trisubstituted alkenes through sequential iron-catalyzed reductive anti-carbozincation of terminal alkynes and base-metal-catalyzed negishi cross-coupling. Chem. Eur. J. 21, 18439-18444 (2015).

46. Lee, J. T. D. \& Zhao, Y. Access to acyclic Z-enediynes by alkyne trimerization: Cooperative bimetallic catalysis using air as the oxidant. Angew. Chem. Int. Ed. 55, 13872-13876 (2016).

47. Rivada-Wheelaghan, O., Chakraborty, S., Shimon, L. J. W., Ben-David, Y. \& Milstein, D. Z-selective (cross)dimerization of terminal alkynes catalyzed by an iron complex. Angew. Chem. Int. Ed. 55, 6942-6945 (2016).

48. Trost, B. M. \& Masters, J. T. Transition metal-catalyzed couplings of alkynes to 1,3-enynes: Modern methods and synthetic applications. Chem. Soc. Rev. 45, 2212-2238 (2016).

49. Wang, N. N. et al. Synergistic rhodium/copper catalysis: Synthesis of 1,3-enynes and $\mathrm{N}$-aryl enaminones. Org. Lett. 18, 1298-1301 (2016).

50. Zhou, Y., Zhang, Y. \& Wang, J. Recent advances in transition-metal-catalyzed synthesis of conjugated enynes. Org. Biomol. Chem. 14, 6638-6650 (2016).

51. Gorgas, N. et al. Stable, yet highly reactive nonclassical iron(II) polyhydride pincer complexes: Z-selective dimerization and hydroboration of terminal alkynes. J. Am. Chem. Soc. 139, 8130-8133 (2017).

52. Yan, Z., Yuan, X. A., Zhao, Y., Zhu, C. \& Xie, J. Selective hydroarylation of 1,3-diynes using a dimeric manganese catalyst: Modular synthesis of Z-enynes. Angew. Chem. Int. Ed. 57, 12906-12910 (2018).

53. Ye, C. et al. Iron-catalyzed dehydrative alkylation of propargyl alcohol with alkyl peroxides to form substituted 1,3-enynes. Org. Lett. 20, 3202-3205 (2018).

54. Cembellín, S., Dalton, T., Pinkert, T., Schäfers, F. \& Glorius, F. Highly selective synthesis of 1,3-enynes, pyrroles, and furans by manganese(I)-catalyzed C-H activation. ACS Catal. 10, 197-202 (2020).

55. Dhungana, R. K., Kc, S., Basnet, P. \& Giri, R. Transition metal-catalyzed dicarbofunctionalization of unactivated olefins. Chem. Rec. 18, 1314-1340 (2018).

56. Derosa, J., Apolinar, O., Kang, T., Tran, V. T. \& Engle, K. M. Recent developments in nickel-catalyzed intermolecular dicarbofunctionalization of alkenes. Chem. Sci. 11, 4287-4296 (2020).

57. Garcia-Dominguez, A., Li, Z. \& Nevado, C. Nickel-catalyzed reductive dicarbofunctionalization of alkenes. J. Am. Chem. Soc. 139, 6835-6838 (2017).

58. Shu, W. et al. Ni-catalyzed reductive dicarbofunctionalization of nonactivated alkenes: Scope and mechanistic insights. J. Am. Chem. Soc. 141, 13812-13821 (2019). 
59. Zhao, X. et al. Intermolecular selective carboacylation of alkenes via nickel-catalyzed reductive radical relay. Nat. Commun. 9, 3488 (2018).

60. Yang, T., Chen, X., Rao, W. \& Koh, M. J. Broadly applicable directed catalytic reductive difunctionalization of alkenyl carbonyl compounds. Chem 6, 738-751 (2020).

61. Wei, X., Shu, W., Garcia-Dominguez, A., Merino, E. \& Nevado, C. Asymmetric Ni-catalyzed radical relayed reductive coupling. J. Am. Chem. Soc. 142, 13515-13522 (2020).

62. Tu, H. Y. et al. Enantioselective three-component fluoroalkylarylation of unactivated olefins through nickelcatalyzed cross-electrophile coupling. J. Am. Chem. Soc. 142, 9604-9611 (2020).

63. Chen, Z., Jiang, H., Wang, A. \& Yang, S. Transition-metal-free homocoupling of 1-haloalkynes: A facile synthesis of symmetrical 1,3-diynes. J. Org. Chem. 75, 6700-6703 (2010).

64. Ping, Y. et al. Ni-catalyzed regio- and enantioselective domino reductive cyclization: One-pot synthesis of 2,3-fused cyclopentannulated indolines. ACS Catal. 9, 7335-7342 (2019).

65. Orsino, A. F., Gutierrez Del Campo, M., Lutz, M. \& Moret, M. E. Enhanced catalytic activity of nickel complexes of an adaptive diphosphine-benzophenone ligand in alkyne cyclotrimerization. ACS Catal. 9, 2458-2481 (2019).

66. Poremba, K. E., Dibrell, S. E. \& Reisman, S. E. Nickel-catalyzed enantioselective reductive cross-coupling reactions. ACS Catal. 10, 8237-8246 (2020).

67. Montgomery, J. Nickel-catalyzed reductive cyclizations and couplings. Angew. Chem. Int. Ed. 43, 3890-3908 (2004).

68. Cheung, C. W., Zhurkin, F. E. \& Hu, X. Z-selective olefin synthesis via iron-catalyzed reductive coupling of alkyl halides with terminal arylalkynes. J. Am. Chem. Soc. 137, 4932-4935 (2015).

69. Zhu, C., Yue, H., Chu, L. \& Rueping, M. Recent advances in photoredox and nickel dual-catalyzed cascade reactions: Pushing the boundaries of complexity. Chem. Sci. 11, 4051-4064 (2020).

70. Allen, L. J., Cabrera, P. J., Lee, M. \& Sanford, M. S. N-acyloxyphthalimides as nitrogen radical precursors in the visible light photocatalyzed room temperature $\mathrm{C}-\mathrm{H}$ amination of arenes and heteroarenes. J. Am. Chem. Soc. 136, 5607-5610 (2014).

71. Huihui, K. M. et al. Decarboxylative cross-electrophile coupling of $N$-hydroxyphthalimide esters with aryl iodides. J. Am. Chem. Soc. 138, 5016-5019 (2016).

72. Gao, Y., Zhang, P., Ji, Z., Tang, G. \& Zhao, Y. Copper-catalyzed cascade radical addition-cyclization halogen atom transfer between alkynes and unsaturated $\alpha$-halogenocarbonyls. ACS Catal. 7, 186-190 (2017).

73. Tsukada, N., Ninomiya, S., Aoyama, Y. \& Inoue, Y. Palladium-catalyzed selective cross-addition of triisopropylsilylacetylene to internal and terminal unactivated alkynes. Org. Lett. 9, 2919-2921 (2007).

74. Lindlar H. \& Dubuis R. Palladium catalyst for partial reduction of acetylenes. Org. synth. 46, 89-92 (1966).

75. Capreti, N. M. \& Jurberg, I. D. Michael addition of soft carbon nucleophiles to alkylidene isoxazol-5-ones: A divergent entry to $\beta$-branched carbonyl compounds. Org. Lett. 17, 2490-2493 (2015).

76. Kálai, T., Hubbell, W. L. \& Hideg, K. Click reactions with nitroxides. Synthesis 2009, 1336-1340 (2009).

77. Alonso, P., Pardo, P., Fontaneda, R., Fananas, F. J. \& Rodriguez, F. Synthesis and applications of cyclohexenyl halides obtained by a cationic carbocyclisation reaction. Chem. Eur. J. 23, 13158-13163 (2017).

\section{Acknowledgements}

This research was supported by the Ministry of Education of Singapore Academic Research Funds Tier 1: R-143-000-

B57-114 (M.J.K.) and Tier 2: R-143-000-A94-112 (Y.Z.).

\section{Author contributions}


Y.J., J.P. and J.J.H.L. developed the catalytic method. M.J.K. and Y.Z. directed the investigations. M.J.K. wrote the manuscript with revisions provided by the other authors.

\section{Competing interests}

The authors declare no competing financial interests. 